This item was submitted to Loughborough's Research Repository by the author.

Items in Figshare are protected by copyright, with all rights reserved, unless otherwise indicated.

\title{
Thermo-behavioural responses to orally applied L-menthol exhibit sex- specific differences during exercise in a hot environment
}

\section{PLEASE CITE THE PUBLISHED VERSION}

https://doi.org/10.1016/j.physbeh.2020.113250

\section{PUBLISHER}

Elsevier BV

VERSION

AM (Accepted Manuscript)

\section{PUBLISHER STATEMENT}

This paper was accepted for publication in the journal Physiology \& Behavior and the definitive published version is available at https://doi.org/10.1016/j.physbeh.2020.113250

\section{LICENCE}

CC BY-NC-ND 4.0

\section{REPOSITORY RECORD}

Parton, Abbie Jayne, Mark Waldron, Tom Clifford, and Owen Jeffries. 2020. "Thermo-behavioural Responses to Orally Applied L-menthol Exhibit Sex-specific Differences During Exercise in a Hot Environment". Loughborough University. https://hdl.handle.net/2134/13258817.v1. 
1 Title: Thermo-behavioural responses to orally applied L-menthol exhibit sex-specific differences during exercise in a hot environment

3

Authors and affiliations:

Parton, Abbie Jayne ${ }^{1}$; Waldron, Mark ${ }^{2,3}$; Clifford, Tom ${ }^{4}$; Jeffries, Owen..$^{1^{*}}$

Institutions:

${ }^{1}$ School of Biomedical, Nutritional and Sport Sciences, Newcastle University, Newcastle Upon Tyne, UK

$11{ }^{2}$ College of Engineering, Swansea University, Swansea, UK.

$12{ }^{3}$ School of Science and Technology, University of New England, Armidale, NSW, Australia.

$13{ }^{4}$ School of Sport, Health and Exercise Sciences, Loughborough University, Loughborough, UK.

* = corresponding author

17 Contact Details for the Corresponding Author:

18 Dr Owen Jeffries

19 School of Biomedical, Nutritional and Sport Sciences,

20 Newcastle University,

21 Newcastle Upon Tyne,

22 NE2 4HH

23 UK

Email: owen.jeffries@newcastle.ac.uk

27 Running head:

28 Keywords: Thermoregulation; Perception; Menthol; Heat; Female; Gender 
33 Aims: This study investigated the efficacy of L-menthol mouth-rinsing on thermal sensation and perceived

34 effort in females and males, using a fixed-rating of perceived exertion (RPE) exercise protocol in a hot environment.

Methods: Twenty-two participants (eleven females, eleven males) completed two trials using a fixed-RPE protocol at an exercise intensity between 'hard' and 'very hard', equating to 16 on the RPE scale at $~ 35^{\circ} \mathrm{C}$. Participants adjusted power output to maintain RPE-16. In a randomised, double-blind, crossover design, L-menthol or a control mouthwash was administered at an orally neutral temperature $\left(\sim 32{ }^{\circ} \mathrm{C}\right)$ prior to exercise and at $10 \mathrm{~min}$ intervals thereafter. Measures of mechanical power output, core temperature, heart rate, perception of thermal sensation and thermal comfort, and whole-body sweat loss are reported.

Results: Thermal sensation was lowered by L-menthol in both sexes $(P<0.05)$, however during exercise this was only maintained for $40 \%$ of the trial duration in females. Thermal comfort did not differ between conditions $(P>0.05)$. No differences in exercise duration were observed compared to control, despite a $\sim 4$ $\%$ and $\sim 6 \%$ increase in male and females respectively. Power output increased by $\sim 6.5 \%$ males $(P=0.039)$ with no difference in females $2.2 \%(P=0.475)$, compared to control. Core temperature, heart rate and whole-body sweat loss was not different between condition or sex.

Conclusions: L-menthol lowered perceptual measures of thermal sensation in females, but did not attenuate a greater rate of rise in thermal sensitivity when exercising in a hot environment, compared to males. Males appeared to adopt a higher risk strategy by increasing power output following L-menthol administration in contrast to a more conservative pacing strategy in females. Therefore, there appear to be sex-specific differences in L-menthol's non-thermal cooling properties and subsequent effects on thermo-behavioural adjustments in work-load when exercising in a hot environment. 
60 There are a number of reported sex differences in thermoregulatory responses to exercise in hot environments (Fox et al., 1969; Gagnon and Kenny, 2012; Shapiro et al., 1980; Smith and Havenith, 2012). In eumenorrheic women, core temperature displays a biphasic rhythm across the menstrual cycle, with $0.4{ }^{\circ} \mathrm{C}$ increase during the post-ovulatory luteal phase (Marshall, 1963) due to a change in thermoregulatory set point (Inoue et al., 2005; Pivarnik et al., 1992; Tenaglia et al., 1999). As a result, the threshold for thermoregulatory effector responses is increased (Inoue et al., 2005; Kolka and Stephenson, 1997; Stachenfeld et al., 2000), and an increase in cardiorespiratory strain has been reported (Janse de Jonge, 2003; Pivarnik et al., 1992). Indeed, heat tolerance is reduced by 6-16 \% during exercise tasks performed in the mid-luteal phase when compared to the early follicular phase (Avellini et al., 1980; De Jonge et al., 2012; Tenaglia et al., 1999). Hormonal contraceptive use is prevalent in females and female athletes (Martin et al., 2018; Rechichi et al., 2009) were suppression of endogenous hormone concentrations inhibits ovulation. However, phase-related changes in core temperature and effector responses are still apparent $\left(\sim 0.15^{\circ} \mathrm{C}\right)$ (Grucza et al., 1993; Lei et al., 2019) and therefore should still be considered when examining strategies to enhance heat tolerance in females using hormonal contraceptives.

Behavioural thermoregulation is the first strategy to defend against a disruption in heat balance in hot environments, secondary to changes in body temperature (Flouris and Schlader, 2015). Subjective responses to physical activity, which include perceived exertion, thermal sensation and sensation of pain, are known to vary in females according to menstrual cycle phase (Gerrett et al., 2014; Hooper et al., 2011; Travlos and Marisi, 1996). In hot conditions, alteration of thermal sensitivity leads to behavioural reductions in exercise intensity following stimulation of peripheral thermoreceptors, which demonstrate regional sensitivity (Nakamura et al., 2008). However, there is clear variation in thermal sensitivity between sexes (Gerrett et al., 2014), with females able to detect warm (Gerrett et al., 2014; Golja et al., 2003; Lautenbacher and Strian, 1991) and cold stimuli (Golja et al., 2003) more strongly than males, independent of changes in body temperature. Higher sensitivity is reported around the head regions with respect to the extremities (Gerrett et al., 2014); however, the oral cavity is one of the most densely innervated parts of the body in terms of peripheral receptors (Haggard and de Boer, 2014). Limited research has been presented on sex differences in oral sensitivity, with one study reporting no differences in relation to sex or phases of the menstrual cycle (Abe et al., 2012). We have recently shown that ice slushy or L-menthol oral mouth-rinsing during advanced thermal stress can extend exercise performance (a conscious behaviour), despite no change in body temperature, in males (Jeffries et al., 2018). Female participants are 
92 recent growth in research into L-menthol's ergogenic properties during exercise in hot and indoor 93 environments has not yet extended to females.

94 When administered orally, L-menthol non-thermally enhances cold sensations in the mouth (Eccles, 1994) 95 and inhibits the perception of warmth (Green, 1986), ultimately leading to a conscious reduction in thermal 96 sensation, which is particularly effective when exercising in a hot environment (Jeffries and Waldron, 97 2019). Fixed-RPE exercise protocols allow instantaneous thermo-behavioural adjustments in work-load to 98 be monitored, whereby individuals can integrate perceptual, peripheral and environmental cues to self99 determine work intensity. We have previously shown that L-menthol can increase work-load and extend 100 exercise time during a fixed-RPE protocol in hot conditions, in males (Flood et al., 2017). Considering the 101 reported greater thermal sensitivity in females, it is unknown if L-menthol may elicit comparable or 102 stronger effects than observed in males. In addition, L-menthol is typically delivered in a solution cooler 103 (19-23 $\left.{ }^{\circ} \mathrm{C}\right)$ (Flood et al., 2017; Mündel and Jones, 2010; Riera et al., 2014; Stevens et al., 2015, 2016) than 104 oral temperature $\left(\sim 36^{\circ} \mathrm{C}\right)$, hence potentiating the thermal cooling capacity of each mouth rinse (Green, 105 1985). Therefore, we delivered L-menthol at a temperature that would be thermally neutral in the mouth 106 to isolate L-menthol's efficacy in modulating perceived thermal sensation and resultant effects on 107 behaviour in both sexes when exercising in the heat.

108 Our aims were to investigate L-menthol mouth-rinsing in males and females using a fixed-RPE exercise 109 protocol in hot conditions. We hypothesised that females would exhibit a reduction in perceived thermal 110 sensitivity following non-thermal cooling provided by orally applied L-menthol that would be equally or 111 more effective in facilitating an increased work-load and extension in task performance as we have 112 previously described for males (Flood et al., 2017). 
117 Twenty-two non-acclimated participants, comprising eleven females (age $=22 \pm 2$ years; body mass $=65.3$

$118 \pm 4.0 \mathrm{~kg}$; stature $=167.6 \pm 4.2 \mathrm{~cm}$; maximal oxygen uptake, $\dot{V} \mathrm{O}_{2 \max }=43.5 \pm 2.9 \mathrm{ml} \cdot \mathrm{min} \cdot \mathrm{kg}^{-1}$ ) and eleven

119 males (age $=20 \pm 1$ years; body mass $=77.7 \pm 8.9 \mathrm{~kg}$; stature $=180.0 \pm 6.0 \mathrm{~cm}$; maximal oxygen uptake,

$120 \dot{V} \mathrm{O}_{2 \max }=53.9 \pm 6.9 \mathrm{ml} \cdot \mathrm{min} \cdot \mathrm{kg}^{-1}$ ) consented to take part in this study. A priori sample size was calculated

121 using G*Power (version 3.1.9.6). Given the effect size $\left(\eta_{p}{ }^{2}=0.896\right.$; (Flood et al., 2017)) we reported

122 previously for differences in power output using an RPE-16 protocol with L-menthol, a sample size of ten

123 was deemed sufficient to identify differences between groups with a statistical power of 0.95 . We recruited

124 eleven participants to account for experimental mortality. Participants engaged in regular physical activity

$125<5$-h per week. None of the participants had visited a hot country in the previous three months, all resided

126 in the UK and experiments were conducted in one block during the winter months of January - March.

127 Participants were instructed to avoid consumption of alcohol or caffeinated products for 24-h before each

128 visit, as well as strenuous exercise 48-h before testing and to arrive fully hydrated. Ethical approval was

129 provided by Newcastle University ethics committee, which was conducted in accordance with the 1964

130 Helsinki declaration.

\section{Study design}

134 A randomised, double-blind, crossover design examined L-Menthol mouth rinse in males and females

135 during exercise in the heat using a fixed RPE protocol (Flood et al., 2017). Randomisation was conducted

136 by generating random numbers for each condition for all participants using online software (Urbaniak and

137 Plous, 2015) and blinding was performed by a person that was not on the research team and all solutions

138 were administered with random letters. Participants were blinded to the original hypothesis of the study

139 and informed that the effect of differing mouth-rinse flavours on exercise in the heat was being 140 investigated. Participants visited the Laboratory on three separate occasions. During visit 1, participants 141 conducted baseline testing to establish maximal oxygen uptake $\left(\dot{V} \mathrm{O}_{2 \max }\right)$ and power output at $\dot{V} \mathrm{O}_{2 \max }$ $142\left(W_{\max }\right)$, as well as being fully familiarised to the experimental protocol. During visits 2 and 3 , the participants 143 completed the fixed-RPE protocol either with L-menthol or control mouth rinse, which were all 144 administered at approximate mouth temperature.

\section{Experimental procedures}


149 All females enrolled in this study were taking hormonal contraceptives (eight: combined oral contraceptive

150 Rigenidon ${ }^{\circledR}$; three: progesterone contraceptive implant Nexplanon $\left.{ }^{\circledR}\right)$. Testing was designed to take place 151 during the quasi early-to-mid follicular phase (approximately day 2-10). In females taking the combined 152 oral contraceptive, where oestrogen and progesterone is downregulated, a quasi-follicular phase was 153 calculated based on day 1 of the 28-day pill regime representing the beginning of the menstrual cycle. 154 Females using progesterone implants, where a reduction in endogenous progesterone but not oestrogen 155 is observed and hormonal fluctuations and menses may occur, we determined the correct phase using the 156 forward counting method, which determines menstrual phases by counting the number of days from the 157 previous onset of menses (Janse de Jonge, 2003). This method has acknowledged limitations due to 158 variable follicular phases, particularly when using progesterone contraceptives and therefore we retrospectively calculated menstrual length by asking participants to report their next onset of menses, 160 after testing was complete. All participants were tested \pm 2 days of their calculated early-to-mid follicular 161 phase. All experimental tests where timetabled to occur during an 8-day period (eg. day 2-10) based on the predicted quasi early-to-mid follicular phase with 72-h between tests.

Preliminary testing

165 Participants reported to the laboratory to conduct preliminary testing consisting of anthropometric 166 measurements and an incremental ramp test. Participants then performed a self-paced warm-up for 5-min 167 and were asked to select a preferred cadence that was standardised throughout the remaining 168 experimental trials. The incremental ramp test began at $100 \mathrm{~W}$, and work-load increased in one-min stages 169 at a rate of $25 \mathrm{~W} \cdot \mathrm{min}^{-1}$ until volitional fatigue. All testing was conducted on an electronically-braked cycle 170 ergometer (Velotron Racermate, USA). Expired gases were analysed using the Douglas bag method. 171 Expired gases were collected by a mouthpiece connected to a 2-way Hans-Rudolph breathing valve (27000 172 series) (Hans Rudolph, inc. USA) and a 2-meter corrugated hose over a collection period $\sim 45$-s. At the end 173 of the collection period, gas fractions $\left(\mathrm{F}_{\mathrm{E}} \mathrm{O}_{2}\right.$ and $\left.\mathrm{F}_{\mathrm{E}} \mathrm{CO}_{2}\right)$ were analysed (Servomex, 5200 MiniMP, UK), 174 volume of expired air (Harvard Apparatus, Kent, UK) and air temperature were measured for calculation 175 of $\dot{V} \mathrm{O}_{2 \max }$ by indirect calorimetry. All values were corrected to reflect standard temperature and pressures. $176 \dot{V} \mathrm{O}_{2 \max }$ was determined as the highest average 30 -s value obtained. RPE was recorded at the end of each 177 1-min stage by pointing to a 15-grade RPE scale held by an investigator. Following a 15-min rest period, 178 two familiarisation exercises were conducted which were subsequently used with the intention of 179 calibrating the participant's RPE-based selection of power output in the main trials. In the first exercise, 180 participants conducted incremental ramp steps in accordance with the power output / RPE relationship 181 derived from the incremental ramp test. The steps followed the order: RPE 11 for 4-min, RPE 13 for 3-min, 182 and RPE 15 for 2-min. Participants were blinded to the RPE and asked to rate their own RPE to aid 183 familiarisation with the RPE scale. The second exercise began at $110 \mathrm{~W}$ and involved participants controlling 
resistance on the ergometer, whilst being blinded to actual power output, in order to achieve an RPE they

185 perceived as equalling RPE-16 over a period of 5-min. The final power output was recorded as the power

186 output at the level of cycling resistance that the participant indicated best represented an RPE-16. The

187 latter test was used to demonstrate the reliability of the participant's ability to select a replicable exercise

188 intensity at the desired RPE across the familiarisation and experimental trials prior to administration of the

189 mouth rinse.

\section{Experimental trials}

192 Participants performed two randomised experimental trials in an environmental heat chamber in 193 temperatures of $34.9 \pm 0.5{ }^{\circ} \mathrm{C}$ and relative humidity $40.6 \pm 2.2 \%$, separated by at least $72-\mathrm{h}$. For each 194 participant, the experimental trials were conducted at the same time of day to eliminate the effect of circadian variation. Euhydration was established prior to exercise by identifying urine osmolality $<715$ mOsm/Kg H $\mathrm{H}_{2} \mathrm{O}$ (Shirreffs and Maughan, 1998) (Pocket Osmochek, Vitech Scientific Ltd, West Sussex, UK) and average hydration was $388.8 \pm 243.5 \mathrm{mOsmols} / \mathrm{kg} \mathrm{H}_{2} \mathrm{O}$, across both conditions. Participants were

198 instrumented with a heart rate chest strap then entered the heat chamber, resting for 10 minutes before 199 baseline measures were recorded. Participants then conducted a standardised warm-up procedure, as 200 outlined previously in the second familiarisation exercise, ramping to an RPE-16 over a 5 min period. Following 5-min of seated rest, participants then started the fixed-RPE protocol.

\section{Fixed-RPE protocol}

204 Participants were instructed to cycle at a power output that was perceived to represent an RPE of 16 on the 15-grade Borg scale (Borg, 1982) and to adjust their power output such that an RPE of 16 was maintained. An RPE of 16 represents a verbal cue of between 'hard' and 'very hard' on the Borg Scale. The highest average 30-s power output achieved during the first 3-min of the fixed RPE trial was recorded and participants exercised until their power output declined to $70 \%$ of this initial value (Flood et al., 2017; Tucker et al., 2006). The trial was stopped when power output fell below this value for 30-s. Standardised feedback every 2-min was given to remind participants to maintain an RPE of 16. Participants were encouraged to constantly reassess whether they were still exercising at RPE-16. They were blinded to distance covered, elapsed time, heart rate, power output.

\section{Measurements}

217 Tympanic temperature was recorded every 6-min as an approximation of core temperature. Based on

218 analysis conducted in our laboratory, tympanic temperature measured with the current device (Braun 
219 Thermoscan IRT 6020, UK) underestimates rectal temperature by $0.5 \pm 0.3^{\circ} \mathrm{C}$ but correlates strongly $\left(R^{2}=\right.$ 220 0.92) across a range of sub-maximal exercise intensities and environmental conditions. Participants 221 recorded semi-nude (males: shorts; females: shorts and sports bra) body mass prior to entering the heat 222 chamber and immediately following the completion of the experimental trial after wiping off sweat with a 223 towel. No water was ingested during exercise in the heat. Heart rate was recorded continuously throughout 224 the trials (Polar T31, UK) transmitting data onto a portable watch (Polar FT7, UK).

\section{Perceptual measures}

227 Participants were thoroughly briefed on the RPE scale during familiarisation sessions before commencing the fixed RPE trials as we have previously reported (please see for full description: Flood et al., 2017). Briefly, participants were instructed to pay close attention to how difficult the exercise felt, combining total exertion, fatigue, and physical stress in the heat, without considering one particular factor such as leg pain, shortness of breath or anticipation of how they might feel several minutes later. In addition, participants where familiarised with the thermal sensation scale and thermal comfort scale. Laminated scales were held in front of the participants during exercise and they were asked to indicate thermal comfort and sensation by pointing to the appropriate point on the scale. Thermal comfort (TC) was recorded on the Bedford 7point analogue scale where $-3=$ "much too cool", 0 = "comfortable", and 3 = "much too warm" (Bedford, 1936). Thermal sensation (TS) was recorded on an adapted ASHRAE 9-point analogue sensation scale where $-4=$ "very cold", 0 = "neutral", and $4=$ "very hot" (Zhang et al., 2004). Subjective ratings were recorded in 1.0 increments every 5 min during the experimental trials.

\section{Mouth rinse formulation}

Participants were given $25 \mathrm{ml}$ solution to rinse 30-s prior to the main fixed RPE trial and at regular 10-min intervals (therefore delivered at -0:30, 9:30 and 19:30 min etc). They were instructed to swill around the mouth for 10-s before spitting into a bowl without swallowing. L-menthol solution was formulated from menthol crystals ( $\geq 99 \%$ food grade L-menthol, Sigma-Aldrich, UK) dissolved in de-ionised water heated to $\sim 50{ }^{\circ} \mathrm{C}$ at a concentration of $0.64 \mathrm{mM}(0.01 \%)$ (Flood et al., 2017). The solution was then stored at $5{ }^{\circ} \mathrm{C}$ for up to 1 month. Prior to use, solutions were aliquoted for mouth-rinse and warmed to ambient laboratory temperature $31.8 \pm 2.3^{\circ} \mathrm{C}$ which was confirmed by a standard thermometer and recorded. A control mouth rinse was made using an apple flavoured non-calorific artificial sweetener, consisting of sucralose (Flood et al., 2017). 
254 All statistical analyses were performed using SPSS (IBM SPSS statistics 22 Inc, USA). Sex differences 255 between conditions were examined by collapsing time due to the statistical power required to conduct 256 three-way analysis. Two-way analysis of variance (ANOVA) for repeated measures were then used to test 257 for within-group effects across time in both conditions for each sex. If sphericity was violated a 258 Greenhouse-Geisser correction was applied. When a significant interaction effect (condition $\mathrm{x}$ time) was 259 reported, post-hoc pair-wise comparisons were made incorporating a Bonferroni adjustment. Magnitude 260 of effect was calculated with partial eta-squared $\left(\eta_{p}{ }^{2}\right)$ according to the following criteria: 0.02 , a small 261 difference; 0.13, a moderate difference; 0.26 a large difference (Cohen, 1988). Differing trial durations 262 meant that power data was normalized with respect to time. Trial duration, peak power and changes in 263 body mass were analysed using a 2-tailed paired sample $t$-test and magnitude of effect calculated (Cohen's $264 d$ ) according to the following criteria: 0.2, a small difference; 0.5, a moderate difference; 0.8 a large 265 difference (Cohen, 1988). Perceptual data, reported on an ordinal scale, was analysed using non266 parametric alternatives. A Friedman test was conducted to assess repeated measures and a Wilcoxon 267 signed-rank test to compare average data between sex. Magnitude of effect calculated by dividing the 268 absolute standardised test $\mathrm{z}$ statistic by the square root of the number of pairs according to the following 269 criteria: 0.1, a small difference; 0.3, a moderate difference; 0.5 a large difference (Cohen, 1988). Data are 270 presented as mean \pm SD, significance was set at $P<0.05$. 
275 Data for time and power output were normally distributed and showed no trial order effect $(P>0.05)$. 276 During the pre-experimental warm-up were participants were instructed to self-select an RPE of 16 over 2773 -min from a starting intensity of $110 \mathrm{~W}$, the final power output selected was not different between condition or sexes $\left(F_{(1,20)}=0.019, P=0.893 ; \eta_{p}{ }^{2}=0.001\right)$, despite an observable $\sim 30 \mathrm{~W}$ average difference between males (L-menthol: $170 \pm 32 \mathrm{~W}$; Control: $170 \pm 25 \mathrm{~W}$ ) and females (L-menthol: $139 \pm 15 \mathrm{~W}$; Control: $141 \pm 22 \mathrm{~W})$. Trial duration was not different between $\operatorname{sex}\left(F_{(1,20)}=1.119, P=0.303 ; \eta_{p}{ }^{2}=0.053\right)$ or condition $\left(F_{(1,20)}=0.070, P=0.794 ; \eta_{p}^{2}=0.003\right)$. However, in males (L-menthol: 34:54 $\pm 10: 27$ min; Control: 33:22 \pm 10:36 $\mathrm{min}$ ) there was a nominal $\sim 4 \%(92-\mathrm{s})$ increase in exercise time in the L-menthol condition and in females (L-menthol: 29:42 \pm 7:43 min; Control: 27:51 \pm 5:52 $\mathrm{min}$ ) a 6 \% (111-s) increase in exercise time in the L-menthol condition.

Average power output across the trial was different between condition $\left(F_{(1,20)}=5.917, P=0.025 ; \eta_{p}^{2}=\right.$ 0.228), however the interaction effect indicated no differences between average power output in each condition and $\operatorname{sex}\left(F_{(1,20)}=1.137, P=0.299 ; \eta_{p}^{2}=0.054\right)$. Power output decreased with time in males $\left(F_{(10,100)}\right.$ $\left.=122.114, P=0.000 ; \eta_{p}{ }^{2}=0.924\right)$ and females $\left(F_{(2.117,21.165)}=11.294, P>0.001 ; \eta_{p}{ }^{2}=0.530\right)$. Across the trial, power output was higher in males in the L-menthol condition (L-menthol: $160 \pm 26 \mathrm{~W}$, Control: $150 \pm 26 \mathrm{~W}$ $(\sim 6.5 \%),\left(F_{(1,10)}=5.018, P=0.039 ; \eta_{p}^{2}=0.334\right)$ with an interaction effect $\left(F_{(10,100)}=2.016, P=0.037, \eta_{p}^{2}=\right.$ 0.168) (Figure 1A). However, in females there was no difference between conditions (L-menthol: $127 \pm 11$ W, Control: $124 \pm 14 \mathrm{~W}(\sim 2.2 \%),\left(F_{(1,10)}=0.552, P=0.475 ; \eta_{p}{ }^{2}=0.052\right)$ and no interaction effect $\left(F_{(2.242,22.4525)}\right.$ $=0.801, P=0.474 ; \eta_{p}^{2}=0.074$ ) (Figure $1 \mathrm{~A}$ ). During the first $10 \%$ of the exercise task, all participants achieved their peak power output which was different between males and females $\left(t_{(10)}=-4.083, P=0.002\right.$, $d=1.44)$. In males, self-selected peak power was $\sim 6 \%$ higher in the L-menthol condition, with 8 out of 11 participants selecting a higher power output $\left(t_{(10)}=-2.247, P=0.048, d=0.38\right)$ (Figure 1B). However, no significant difference in peak power ( $2 \%)$ was observed for females $\left(t_{(10)}=-0.627, P=0.545, d=0.15\right)$ (Figure 1B). 
Perceptual measures of thermal sensation increased with time in all conditions for males and females $(P<$ 0.001). However, when collapsed for time there were differences between males and females $(z=-2.357$,

$310 P=0.018, d=0.71$ ), with males reporting on average $\sim 0.6$ points lower on the scale for thermal sensation across both trials. In males, thermal sensation was lowered in the L-menthol condition across the entire trial, except at the 18-min time point (Start $-0.81(z=-2.714 ; P=0.007 ; d=0.82), 6-\min -0.45(z=-2.236 ; P$

$313=0.025 ; d=0.67), 12-\min -0.50(z=-2.049 ; P=0.04 ; d=0.61), 18-\min -0.45(z=-1.492 ; P=0.136 ; d=0.44$,

314 End $-0.63(z=-1.897 ; P=0.05, d=0.60)$ ) (Figure $2 \mathrm{~A})$. In females, thermal sensation was lower only across 315 the first 12-min of exercise in the L-menthol condition (Start $-0.45(z=-1.833 ; P=0.050 ; d=0.55), 6-\mathrm{min}$ $3160.41(z=-2.121 ; P=0.034 ; d=0.64), 12-\min -0.38(z=-1.667 ; P=0.048 ; d=0.50), 18-\min -0.05(z=-0.333$; $317 P=0.739 ; d=0.10)$, End $-0.18(z=-0.973 ; P=0.330 ; d=0.29)$ (Figure 2B). The rate at which thermal 318 sensation increased across the first 18-min of the exercise trials was faster in females (L-menthol 0.12 319 units $/ \mathrm{min}^{-1}, \mathrm{R}^{2}=0.87$; Control 0.13 units $/ \mathrm{min}^{-1}, \mathrm{R}^{2}=0.94$ ) compared to males (L-menthol 0.07 units $/ \mathrm{min}^{-1}$, $320 \mathrm{R}^{2}=0.93$; Control 0.08 units $\left./ \mathrm{min}^{-1}, \mathrm{R}^{2}=0.92\right),\left(t_{(10)}=-2.294, P=0.045, d=0.97\right)$. Thermal comfort increased on the scale, denoting greater discomfort, across time in all conditions for males and females $(P<0.001)$. However, there were no differences at any time point for L-menthol and control conditions or between sex $(P>0.05)$ (Figure $2 \mathrm{C} \& \mathrm{D})$.

Core temperature after the standardized warm-up was not different between conditions in males ( $\mathrm{L}$ menthol: $36.9 \pm 0.4{ }^{\circ} \mathrm{C}$; Control: $36.9 \pm 0.3{ }^{\circ} \mathrm{C}$ ) and females (L-menthol: $37.1 \pm 0.4{ }^{\circ} \mathrm{C}$; Control: $37.2 \pm 0.4{ }^{\circ} \mathrm{C}$ ) $(P>0.05)$. Core temperature increased with time in males $\left(F(4,40)=4.038, P<0.001 ; \eta_{p}{ }^{2}=0.905\right)$ and females $\left(F_{(1.53,15.33)}=30.40, P<0.001 ; \eta_{p}{ }^{2}=0.752\right)$ but with no difference between condition for males $(F$ $\left.(1,10)=0.067, P=0.801 ; \eta_{p}^{2}=0.007\right)$ and females $\left(F_{(1,10)}=2.740, P=0.129 ; \eta_{p}{ }^{2}=0.215\right)$ (Figure $\left.3 \mathrm{~A} \& \mathrm{~B}\right)$. Heart rate increased with time in males $\left(F_{(1.25,12.46)}=223.78, P<0.001 ; \eta_{p}{ }^{2}=0.957\right)$ and females $F_{(1.78,17.79)}$ $\left.=371.11, P<0.001 ; \eta_{p}^{2}=0.974\right)$ but with no difference between conditions for males $\left(F_{(1,10)}=0.018, P=\right.$ $\left.0.897 ; \eta_{p}{ }^{2}=0.002\right)$ and females $\left(F_{(1,10)}=0.001, P=0.992 ; \eta_{p}{ }^{2}=0.030\right)$ (Figure $\left.3 \mathrm{C} \& D\right)$. The change in body mass was not different between pre-to-post for the exercise task in males (Pre: $0.69 \pm 0.3 \mathrm{~kg}$; Post: $0.68 \pm$ $0.2 \mathrm{~kg})\left(t_{(10)}=0.126, P=0.902, d=0.04\right)$ and females (Pre: $0.36 \pm 0.1 \mathrm{~kg}$; Post: $\left.0.39 \pm 0.2 \mathrm{~kg}\right)\left(t_{(10)}=-0.582\right.$, $P=0.574, d=0.24)$. There were no differences between $\operatorname{sex}\left(F_{(1,20)}=0.179, P=0.179 ; \eta_{p}^{2}=0.676\right)$. 
345 The aims of this study were to investigate the efficacy of L-menthol mouth-rinsing in males and females

346 using a fixed-RPE exercise protocol in a hot environment. Oral application of L-menthol lowered perceptual 347 measures of thermal sensation in males, but in females was only effective in the early stages of exercise in

348 the heat. Females exhibited a faster rate of rise in reported thermal sensation in both conditions, when 349 compared to males. Self-selected power output and exercise duration did not differ in females between 350 the L-menthol and control condition (although exercise duration was increased 6 \%). In contrast, males showed a $6.5 \%$ increase in power output and a $4 \%$ increase in exercise duration in the L-menthol trial, replicating our previous findings (Flood et al., 2017). This refutes our primary hypothesis that L-menthol would be equally or more effective in females at reducing thermal sensation and facilitating a comparable increase in exercise work-load, to males. Consistent with the 'non-thermal' mechanistic basis of Lmenthol's cooling effects (Jeffries and Waldron, 2019), there were no changes in core temperature, heart rate or sweat loss between conditions, despite the reported differences in thermal perception and performance.

Research examining sex-specific differences in thermal sensitivity tends to be largely confined to males. In the present study we tested male and female participants and controlled for potential differences in thermoregulation ascribed to the menstrual cycle (De Jonge et al., 2012; Marshall, 1963) by testing females during a calculated quasi-follicular phase. Baseline measures in both sexes confirmed that there were no differences in core temperature and no differences in the rise in core temperature during exercise (Figure 3 A\&B). Thermal sensitivity encompasses the perceived intensity of temperature being sensed by the individual (Gagge et al., 1967). Psychological strategies that are effective at reducing thermal sensitivity have been successful in extending exercise tolerance in the heat (Cheung, 2010; Flouris and Schlader, 2015). We utilised a non-thermal cooling L-menthol mouth-rinse which was effective at reducing thermal sensation across the majority of the exercise test in males. However a significantly smaller reduction in thermal sensation was observed in females, indicating sex-specific differences in L-menthol's effectiveness during exercise in a hot environment. Indeed, L-menthol induced reductions in thermal sensitivity were only observed over the first 12 minutes of exercise in females. During exercise, the rise in perceived thermal sensation was faster in females than in males, reflecting a greater thermal sensitivity which did not differ between condition. That L-menthol was unable to modify this increase in thermal sensation in both sexes supports a possible reduced potency with subsequent administration that we have noted before (Flood et al., 2017). However, thermal sensitivity is also known to decrease during exercise (Gerrett et al., 2015; Ouzzahra et al., 2012), due to a reduction in transmission of sensory information along afferent fibres via exercise-induced analgesia (EIA) (Koltyn, 2000), therefore transmission of thermal sensory information may be reduced. Limited research exists investigating sex-specific differences in EIA with only one study 
378 supporting no difference between males and females (tested in the follicular phase of the menstrual cycle)

379 (Koltyn et al., 2014), and further work is needed.

380 Thermo-behavioural adjustments in work-load during the trials, enabled changes in perceived exertion to

381 be observed using the fixed-RPE protocol. Males voluntarily adopted a higher power output ( 6.5\%) after

382 rinsing with L-menthol which supported our previous observations ( $4 \%$ ) (Flood et al., 2017). However, in

383 females, no difference in power output (>2\%) following L-menthol-rinsing was observed. This was not

384 anticipated in our initial hypothesis. That a lowering of thermal sensation was reported across both sexes

385 in the early stages of the exercise trial suggests that the effectiveness of L-menthol in inducing non-thermal

386 cooling cannot explain this discrepancy. Despite no significant changes, exercise time was extended in

387 males ( $4 \%$ ) and females ( $6 \%)$. It is also unclear how L-menthol may have extended exercise performance

388 in females compared to control conditions when any change in thermal sensation had dissipated beyond

$389 \sim 40 \%$ of the exercise trial. Females are reported use thermal behaviour (such as modification of work-

390 load) to a greater extent than males during exercise (Vargas et al., 2019) which may suggest that a more

391 conservative pacing strategy was adopted. We have also previously proposed that L-menthol could act as

392 a potential distractor to moderately uncomfortable stimuli, such as exercise in a hot environment,

393 irrespective of its cooling properties (Jeffries et al., 2018), which is possible. It should be noted that exercise

394 duration is an arbitrary measure of performance when using a fixed-RPE protocol and should be carefully

395 interpreted. In females, it is possible that by not increasing power output, despite reporting a reduction in

396 thermal sensation, exercise duration could be extended by consciously adopting a more conservative

397 pacing strategy. If we approximate energy utilised (work done) by multiplying exercise duration (s) by

398 power $(\mathrm{J} / \mathrm{s})$ across participants, in the L-menthol trial total work done was increased by $7 \%$ in males and

$3998 \%$ in females relative to the control trial. Therefore, the pacing strategy adopted by males in the L-menthol

400 condition was inherently more aggressive by selecting a higher power output and yet this did not extend

401 total work done beyond the more conservative strategy adopted by females. Typically, males exhibit

402 different self-pacing strategies when compared to females. In repeated sprint study designs, males self-

403 pace at higher exercise intensities, achieve higher total work and show greater power decrements than

404 women, despite comparable cardiovascular strain (Billaut and Bishop, 2012; Panissa et al., 2016). The noted

405 increase in fatigue is likely to be a consequence of their greater absolute initial sprint performance, rather

406 than a sex-specific difference in fatigue (Billaut and Bishop, 2012). Behaviourally, in the case of decision

407 making, males also appear to adopt a higher risk strategy based on physical fitness, or an alteration in

408 motivation to perform exercise, compared to females (Deaner et al., 2015). When examining competition

409 data, sex differences in marathon pacing in non-elites was larger for males in the 2007 Chicago marathon

410 which was hot $\left(27^{\circ} \mathrm{C}\right)$ when compared to the 2009 Chicago marathon which was cool $\left(3^{\circ} \mathrm{C}\right)$, illustrating the

411 greater propensity for a risky pacing strategy, despite unfavourable environmental conditions (Deaner et

412 al., 2015). 
413 Our findings refute our initial hypothesis that greater thermal sensitivities in females (Gerrett et al., 2014, 414 2015) and greater sensitivity to cold stimuli when compared to males (Gerrett et al., 2015) would 415 potentiate L-menthol's effect. The oral cavity is one of the most densely innervated parts of the body in 416 terms of peripheral receptors (Haggard and de Boer, 2014). Mouth-rinsing with L-menthol activates 417 peripheral TRPM8 thermoreceptors on the oral mucosa transmitting information via the trigeminal system 418 which mediates sensations such as burning, cooling and tingling (Laska et al., 1997). Despite reported sex419 related differences in chemosensation, examination of irritants, including menthol, have failed to report 420 sex-specific differences in trigeminal sensitivity (Ohla and Lundström, 2013). Psychophysical tests have 421 identified that these differences may be due to differing cognitive appraisal between the sexes, therefore 422 altering subjective perception (Lundström et al., 2005; Ohla and Lundström, 2013). However, in this study 423 females reported a smaller reduction in thermal sensation following L-menthol mouth-rinsing, than males, which then dissipated. In thermally challenging environments, females tend to be more sensitive to warm 425 stimuli than males and perceive a thermal stimulus to be hotter (Gerrett et al., 2014, 2015). Therefore the 426 hot environment may have been perceived as a greater thermal threat thereby reducing or de-prioritising 427 L-menthol's perceived cooling properties. At present it is clear that further research is required to 428 understand these sex differences in behavioural thermoregulation.

429 Although not a primary aim of this study we administered L-menthol in a thermally neutral solution 32 $430{ }^{\circ} \mathrm{C}$. This was important to experimentally establish L-menthol's efficacy when oral cooling facilitated by the 431 delivery solution was removed. We and others have administered L-menthol in oral rinses between 19-23 $432{ }^{\circ} \mathrm{C}$, in males (Flood et al., 2017; Mündel and Jones, 2010; Riera et al., 2014; Stevens et al., 2015, 2016) and 433 one study at $\sim 40{ }^{\circ} \mathrm{C}$ (Gibson et al., 2019). Perceived sensation of cold in the oral cavity reaches zero at $\sim 32$ $434{ }^{\circ} \mathrm{C}$ (the cold threshold) with perception beginning to shift to warmth as liquid temperature increases above $435 \sim 35^{\circ} \mathrm{C}$ (the warmth threshold), despite oral temperature being $\left(\sim 36^{\circ} \mathrm{C}\right)$ (Green, 1986). Indeed L-menthol 436 solutions below oral temperature have been suggested to feel cooler than water of the same temperature 437 (Green, 1985). These have been demonstrated to potentiate exercise performance in hot humid conditions 438 following neutral $\left(23^{\circ} \mathrm{C}\right)$, cold $\left(3^{\circ} \mathrm{C}\right)$ and ice-slushy $\left(-1{ }^{\circ} \mathrm{C}\right)$ beverage ingestion during a $20-\mathrm{km}$ time-trial 439 (Riera et al., 2014). Therefore it was important to achieve oral temperature neutrality and therefore 440 solutions were administered at $\sim 32{ }^{\circ} \mathrm{C}$ to isolate L-menthol's true non-thermal cooling properties. We can 441 confirm, as previously discussed, in males L-menthol was equally effective in enhancing exercise 442 performance in the heat when delivered at $\sim 32{ }^{\circ} \mathrm{C}$ when compared to our previous study $\sim 19{ }^{\circ} \mathrm{C}$ using 443 identical protocols and ambient conditions albeit a different participant group (Flood et al., 2017). 444 Unfortunately we are unable to make the same comparisons in females. 
447 The females enrolled in this study all used hormonal contraception. Investigations into oral contraceptive 448 users have reported that a phase-related elevation in core temperature $\left(\sim 0.15^{\circ} \mathrm{C}\right)$ and concomitant 449 increase in threshold effector responses is maintained during active and passive heating (Lei et al., 2019). 450 In this study, to eliminate potential effects of the menstrual cycle and to primarily establish whether L451 menthol can modulate exercise performance in the heat we tested females in the quasi-follicular phase of 452 the menstrual cycle in contraceptive users. Eight out of eleven females in this study used the oral-combined 453 contraceptive and three used a progesterone implant contraceptive. There are clear limitations with this 454 combined approach as different contraceptive methods lead to fluctuations in hormone levels (Elliott-Sale 455 et al., 2013); however, we attempted to test only in the predicted and quasi-follicular phase. Blood 456 hormonal confirmation should be sought in future. However, this highlights some interesting future 457 questions regarding L-menthol's effectiveness during different cycle phases of the menstrual cycle, 458 particularly in females not using contraceptives where oscillations in body temperature could modulate L459 menthol's efficacy, particularly in hot conditions. We also acknowledge the limitations with using tympanic 460 temperature to inform changes in core temperature. Core temperature was not a primary outcome 461 measure in this study and we have successfully shown no change in core temperature in a previous study 462 that utilised the same experimental design (Flood et al., 2017), however future studies in combination with 463 hormonal analysis should seek to measure core temperature more accurately.

\section{Conclusion}

465 In summary, L-menthol lowered perceptual measures of thermal sensation during the early stages of 466 exercise in a hot environment in females, but did not attenuate a faster rate of rise in perceived thermal 467 sensation in both conditions when compared to males. Following administration of L-menthol males 468 adopted a higher risk strategy during exercise by increasing power output, however exercise duration was 469 not significantly extended beyond control. Instead females appeared to adopt a more conservative pacing 470 strategy and did not increase power output over control. In conclusion, L-menthol's non-thermal cooling 471 properties and the subsequent modifications of exercise intensity described in males may not be the same 472 in females. Therefore, there appear to be sex-specific differences in L-menthol's non-thermal cooling 473 properties and subsequent effects on thermo-behavioural adjustments in work-load when exercising in a 474 hot environment. 
Abe, M., Uchida, Y., and Toda, S. (2012). Trial of the measurement of the thermal sensitivity in the oral mucosa and lips and lips neighbourhood of the young fellow. Japanese J. Sens. Eval. 16, 43-50.

480

Avellini, B. A., Kamon, E., and Krajewski, J. T. (1980). Physiological responses of physically fit men and women to acclimation to humid heat. J. Appl. Physiol. 49, 254-261. doi:10.1152/jappl.1980.49.2.254.

Bedford, T. (1936). "The warmth factor in comfort at work: a physiological study of heating and ventilation.," in Industrial Health Research Board (London: HMSO).

Billaut, F., and Bishop, D. J. (2012). Mechanical work accounts for sex differences in fatigue during repeated sprints. Eur. J. Appl. Physiol. 112, 1429-1436. doi:10.1007/s00421-011-2110-1.

Borg, G. A. (1982). Psychophysical bases of perceived exertion. Med. Sci. Sports Exerc. 14, 377-381. doi:10.1249/00005768-198205000-00012.

Cheung, S. S. (2010). Interconnections between thermal perception and exercise capacity in the heat. Scand. J. Med. Sci. Sport. 20, 53-59. doi:10.1111/j.1600-0838.2010.01209.x.

Cohen, J. (1988). Statistical power analysis for the behavioral sciences. 2nd ed. New York: Erlbaum, Hillsdale.

Costello, J. T., Bieuzen, F., and Bleakley, C. M. (2014). Where are all the female participants in Sports and Exercise Medicine research? Eur. J. Sport Sci. 14, 847-851. doi:10.1080/17461391.2014.911354.

De Jonge, J., Thompson, M., Chuter, V., Silk, L., and Thom, J. (2012). Exercise performance over the menstrual cycle in temperate and hot, humid conditions. Med. Sci. Sports Exerc. 44, 2190-2198. doi:10.1249/MSS.0b013e3182656f13.

Deaner, R. O., Carter, R. E., Joyner, M. J., and Hunter, S. K. (2015). Men are more likely than women to slow in the marathon. Med. Sci. Sports Exerc. 47, 607-616. doi:10.1249/MSS.0000000000000432.

Eccles, R. (1994). Menthol and Related Cooling Compounds. J. Pharm. Pharmacol. 46, 618-630. doi:10.1111/j.2042-7158.1994.tb03871.x.

Elliott-Sale, K. J., Smith, S., Bacon, J., Clayton, D., McPhilimey, M., Goutianos, G., et al. (2013). Examining the role of oral contraceptive users as an experimental and/or control group in athletic performance studies. Contraception 88, 408-412. doi:10.1016/j.contraception.2012.11.023.

Flood, T. R., Waldron, M., and Jeffries, O. (2017). Oral L-menthol reduces thermal sensation, increases workrate and extends time to exhaustion, in the heat at a fixed rating of perceived exertion. Eur. J. Appl. Physiol. 117, 1501-1512. doi:10.1007/s00421-017-3645-6. 
Flouris, A. D., and Schlader, Z. J. (2015). Human behavioral thermoregulation during exercise in the heat. Scand. J. Med. Sci. Sports 25 Suppl 1, 52-64. doi:10.1111/sms.12349.

508

509

510

511

512

513

514

515

516

517

518

519

520

521

522

523

524

525

526

527

528

529

530

531

532

533

534

535

Fox, R. H., Lofstedt, B. E., Woodward, P. M., Eriksson, E., and Werkstrom, B. (1969). Comparison of thermoregulatory function in men and women. J. Appl. Physiol. 26, 444-453. doi:10.1152/jappl.1969.26.4.444.

Gagge, A. P., Stolwijk, J. A., and Hardy, J. D. (1967). Comfort and thermal sensations and associated physiological responses at various ambient temperatures. Environ. Res. 1, 1-20. doi:10.1016/00139351(67)90002-3.

Gagnon, D., and Kenny, G. P. (2012). Sex differences in thermoeffector responses during exercise at fixed requirements for heat loss. J. Appl. Physiol. 113, 746-757. doi:10.1152/japplphysiol.00637.2012.

Gerrett, N., Ouzzahra, Y., Coleby, S., Hobbs, S., Redortier, B., Voelcker, T., et al. (2014). Thermal sensitivity to warmth during rest and exercise: a sex comparison. Eur. J. Appl. Physiol. 114, 1451-1462. doi:10.1007/s00421-014-2875-0.

Gerrett, N., Ouzzahra, Y., Redortier, B., Voelcker, T., and Havenith, G. (2015). Female thermal sensitivity to hot and cold during rest and exercise. Physiol. Behav. 152, 11-19. doi:10.1016/j.physbeh.2015.08.032.

Gibson, O. R., Wrightson, J. G., and Hayes, M. (2019). Intermittent sprint performance in the heat is not altered by augmenting thermal perception via L-menthol or capsaicin mouth rinses. Eur. J. Appl. Physiol. 119, 653-664. doi:10.1007/s00421-018-4055-0.

Golja, P., Tipton, M., and Mekjavic, I. (2003). Cutaneous thermal thresholds: the reproducibility of their measurements and the effect of gender. J. Therm. Biol. 28, 341-346.

Green, B. G. (1985). Menthol modulates oral sensations of warmth and cold. Physiol. Behav. 35, 427-434.

Green, B. G. (1986). Menthol inhibits the perception of warmth. Physiol. Behav. 38, 833-838. doi:10.1016/0031-9384(86)90050-8.

Grucza, R., Pekkarinen, H., Titov, E., Kononoff, A., and Hänninen, O. (1993). Influence of the menstrual cycle and oral contraceptives on thermoregulatory responses to exercise in young women. Eur J Appl Physiol Occup Physiol. 76, 279-85.

Haggard, P., and de Boer, L. (2014). Oral somatosensory awareness. Neurosci. Biobehav. Rev. 47, 469-484. doi:10.1016/j.neubiorev.2014.09.015.

Hooper, A. E. C., Bryan, A. D., and Eaton, M. (2011). Menstrual cycle effects on perceived exertion and pain during exercise among sedentary women. J. Womens. Health (Larchmt). 20, 439-446. 
doi:10.1089/jwh.2010.2042.

537

538

539

540

542

543

544

545

546

547

548

549

550

551

552

553

554

555

556

557

558

559

560

561

562

563

564

Inoue, Y., Tanaka, Y., Omori, K., Kuwahara, T., Ogura, Y., and Ueda, H. (2005). Sex- and menstrual cycle-related differences in sweating and cutaneous blood flow in response to passive heat exposure. Eur. J. Appl. Physiol. 94, 323-332. doi:10.1007/s00421-004-1303-2.

Janse de Jonge, X. (2003). Effects of the menstrual cycle on exercise performance. Sports Med. 33, 833-851. doi:10.2165/00007256-200333110-00004.

Jeffries, O., Goldsmith, M., and Waldron, M. (2018). L-Menthol mouth rinse or ice slurry ingestion during the latter stages of exercise in the heat provide a novel stimulus to enhance performance despite elevation in mean body temperature. Eur. J. Appl. Physiol. doi:10.1007/s00421-018-3970-4.

Jeffries, O., and Waldron, M. (2019). The effects of menthol on exercise performance and thermal sensation: A meta-analysis. J. Sci. Med. Sport 22, 707-715. doi:10.1016/j.jsams.2018.12.002.

Kolka, M. A., and Stephenson, L. A. (1997). Effect of luteal phase elevation in core temperature on forearm blood flow during exercise. J. Appl. Physiol. 82, 1079-1083. doi:10.1152/jappl.1997.82.4.1079.

Koltyn, K. F. (2000). Analgesia following exercise: a review. Sports Med. 29, 85-98. doi:10.2165/00007256200029020-00002.

Koltyn, K. F., Brellenthin, A. G., Cook, D. B., Sehgal, N., and Hillard, C. (2014). Mechanisms of exercise-induced hypoalgesia. J. Pain 15, 1294-1304. doi:10.1016/j.jpain.2014.09.006.

Laska, M., Distel, H., and Hudson, R. (1997). Trigeminal perception of odorant quality in congenitally anosmic subjects. Chem. Senses 22, 447-456. doi:10.1093/chemse/22.4.447.

Lautenbacher, S., and Strian, F. (1991). Sex differences in pain and thermal sensitivity: the role of body size. Percept. Psychophys. 50, 179-183. doi:10.3758/bf03212218.

Lei, T.-H., Cotter, J. D., Schlader, Z. J., Stannard, S. R., Perry, B. G., Barnes, M. J., et al. (2019). On exercise thermoregulation in females: interaction of endogenous and exogenous ovarian hormones. J. Physiol. 597, 71-88. doi:10.1113/JP276233.

Lundström, J. N., Frasnelli, J., Larsson, M., and Hummel, T. (2005). Sex differentiated responses to intranasal trigeminal stimuli. Int. J. Psychophysiol. 57, 181-186. doi:10.1016/j.ijpsycho.2005.01.003.

Marshall, J. (1963). Thermal Changes in the Normal Menstrual Cycle. Br. Med. J. 1, 102-104. doi:10.1136/bmj.1.5323.102.

Martin, D., Sale, C., Cooper, S. B., and Elliott-Sale, K. J. (2018). Period Prevalence and Perceived Side Effects of 

926-932. doi:10.1123/ijspp.2017-0330.

Mündel, T., and Jones, D. A. (2010). The effects of swilling an I(-)-menthol solution during exercise in the heat. Eur. J. Appl. Physiol. 109, 59-65. doi:10.1007/s00421-009-1180-9.

Nakamura, M., Yoda, T., Crawshaw, L. I., Yasuhara, S., Saito, Y., Kasuga, M., et al. (2008). Regional differences in temperature sensation and thermal comfort in humans. J. Appl. Physiol. 105, 1897-1906. doi:10.1152/japplphysiol.90466.2008.

Ohla, K., and Lundström, J. N. (2013). Sex differences in chemosensation: Sensory or emotional? Front. Hum. Neurosci. 7, 1-11. doi:10.3389/fnhum.2013.00607.

Ouzzahra, Y., Havenith, G., and Redortier, B. (2012). Regional distribution of thermal sensitivity to cold at rest and during mild exercise in males. J. Therm. Biol. 37, 517-523.

Panissa, V. L. G., Julio, U. F., Franca, V., Lira, F. S., Hofmann, P., Takito, M. Y., et al. (2016). Sex-Related Differences in Self-Paced All Out High-Intensity Intermittent Cycling: Mechanical and Physiological Responses. J. Sports Sci. Med. 15, 372-378.

Pivarnik, J. M., Marichal, C. J., Spillman, T., and Morrow, J. R. J. (1992). Menstrual cycle phase affects temperature regulation during endurance exercise. J. Appl. Physiol. 72, 543-548. doi:10.1152/jappl.1992.72.2.543.

Rechichi, C., Dawson, B., and Goodman, C. (2009). Athletic performance and the oral contraceptive. Int. J. Sports Physiol. Perform. 4, 151-162. doi:10.1123/ijspp.4.2.151.

Riera, F., Trong, T. T., Sinnapah, S., and Hue, O. (2014). Physical and perceptual cooling with beverages to increase cycle performance in a tropical climate. PLoS One 9, e103718. doi:10.1371/journal.pone.0103718.

Shapiro, Y., Pandolf, K. B., Avellini, B. A., Pimental, N. A., and Goldman, R. F. (1980). Physiological responses of men and women to humid and dry heat. J. Appl. Physiol. 49, 1-8. doi:10.1152/jappl.1980.49.1.1.

Shirreffs, S. M., and Maughan, R. J. (1998). Osmolality and conductivity as markers of hydration status. Med. Sci. Sports Exerc. 30, 1598-1602. doi:10.1097/00005768-199811000-00007.

Smith, C. J., and Havenith, G. (2012). Body mapping of sweating patterns in athletes: a sex comparison. Med. Sci. Sports Exerc. 44, 2350-2361. doi:10.1249/MSS.0b013e318267b0c4. 

performance and thermal sensation in the heat are improved with menthol mouth rinse but not ice slurry ingestion. Scand. J. Med. Sci. Sport., 1-8. doi:10.1111/sms.12555.

Stevens, C. J., Thoseby, B., Sculley, D. V, Callister, R., Taylor, L., and Dascombe, B. J. (2016). Running performance and thermal sensation in the heat are improved with menthol mouth rinse but not ice slurry ingestion. Scand. J. Med. Sci. Sports 26, 1209-1216. doi:10.1111/sms.12555.

601

Tenaglia, S. A., McLellan, T. M., and Klentrou, P. P. (1999). Influence of menstrual cycle and oral contraceptives

602 on tolerance to uncompensable heat stress. Eur. J. Appl. Physiol. Occup. Physiol. 80, 76-83.

603 doi:10.1007/s004210050561.

604

Travlos, A. K., and Marisi, D. Q. (1996). Perceived exertion during physical exercise among individuals high and low in fitness. Percept. Mot. Skills 82, 419-424. doi:10.2466/pms.1996.82.2.419.

Tucker, R., Marle, T., Lambert, E. V, and Noakes, T. D. (2006). The rate of heat storage mediates an anticipatory reduction in exercise intensity during cycling at a fixed rating of perceived exertion. J. Physiol. 574, 905915. doi:10.1113/jphysiol.2005.101733.

Urbaniak, G. C., and Plous, S. (2015). Research randomizer (version 4.0) [computer software]. Available at: http://www.randomizer.org/.

611 Vargas, N. T., Chapman, C. L., Sackett, J. R., Johnson, B. D., Gathercole, R., and Schlader, Z. J. (2019). Thermal Behavior Differs between Males and Females during Exercise and Recovery. Med. Sci. Sports Exerc. 51, 141-152. doi:10.1249/MSS.0000000000001756.

614 Zhang, H., Huizenga, C., Arenas, E., and Wang, D. (2004). Thermal sensation and comfort in transient non615 uniform thermal environments. Eur. J. Appl. Physiol. 92, 728-733. doi:10.1007/s00421-004-1137-y.

616 


\section{Figure legends}

619

620

621 Figure 1. A. Power output against trial duration expressed as a percentage of final time for males and

622 females. Error bars have been removed for clarity. Asterisk denotes significant difference in average power

623 between conditions in males $(P=0.039)$. B. Peak power output selected during the fixed-RPE trial for males

624 and females. Solid lines indicate an increase and dashed lines a decrease between conditions. Asterisk

625 denotes significant difference in peak power between conditions in males $(P=0.048)$. Conditions are

626 indicated by colour, L-menthol (white) and control (black) and sex indicated on figures. All individual data

627 is shown $(\mathrm{n}=22)$.

628

629 Figure 2. A. Thermal sensation as reported during the fixed-RPE trial for males and B. females. C. Thermal 630 comfort as reported during the fixed-RPE trial for males and D. females. Conditions are indicated by colour, 631 L-menthol (white) and control (black) and sex indicated on figures. All data are shown as mean \pm SD, $(n=$ 632 22). Asterisk denotes significant difference between conditions at respective time points $(P<0.05)$.

633

634 Figure 3. A. Core temperature during the fixed-RPE trial for males and B. females. C. Heart rate during the 635 fixed-RPE trial for males and D. females. Conditions are indicated by colour, L-menthol (white) and control 636 (black) and sex indicated on figures. All data are shown as mean $\pm S D,(n=22)$.

637

638 
Declarations of interest: none

640

641 Funding: Authors received no funding for this study

642 
643 Figure 1

644
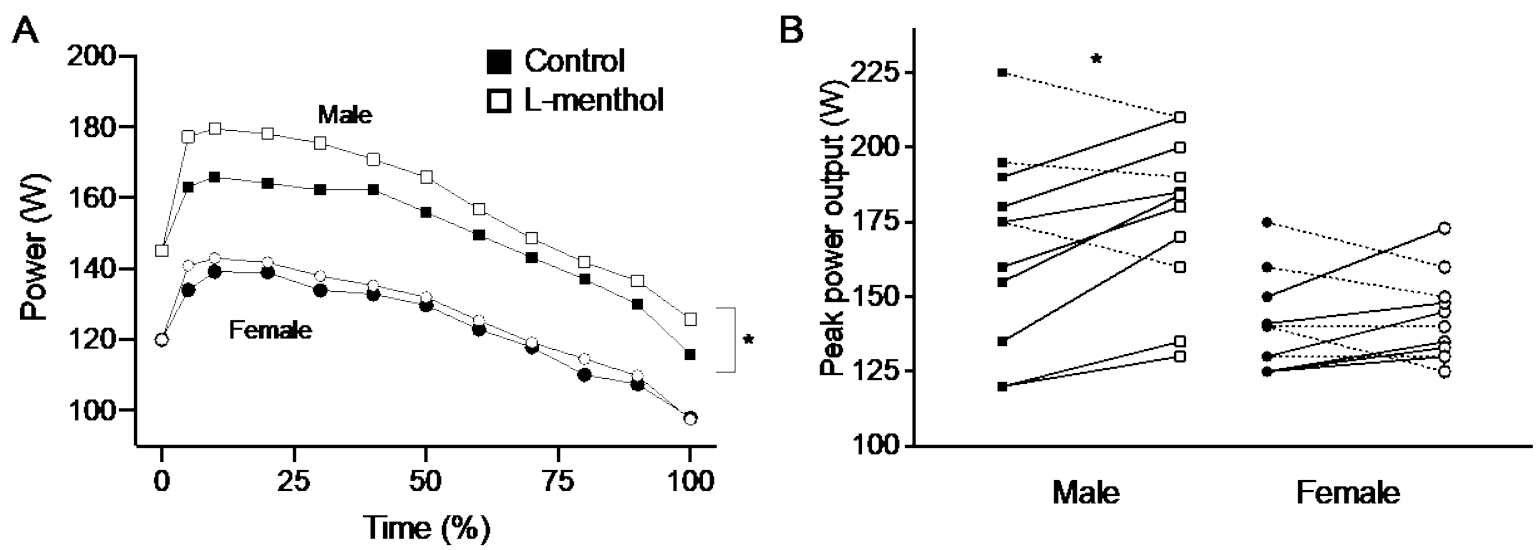

645

646

647 
Figure 2

649

Thermal sensation

A

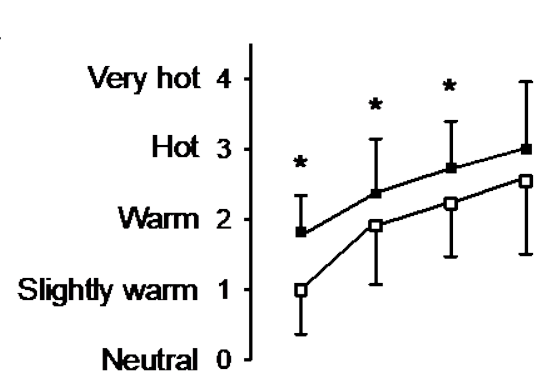

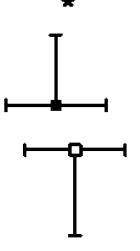

Thermal comfort$$
\text { C }
$$

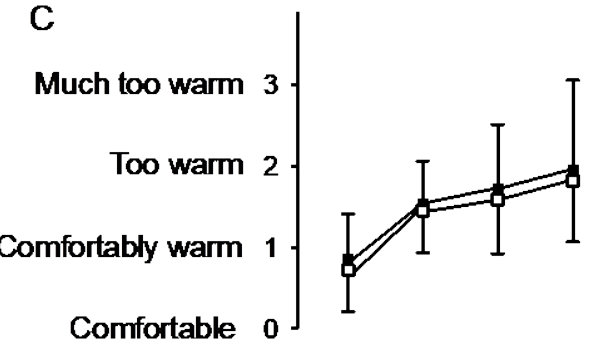

B

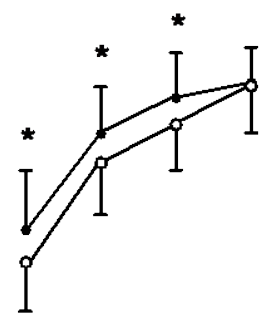<smiles>CC1=C(C)C(C)(C)C1</smiles>

Control

口 L-menthol

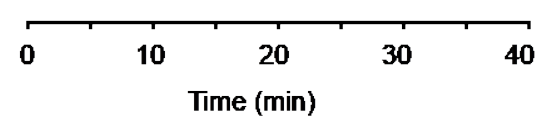

D
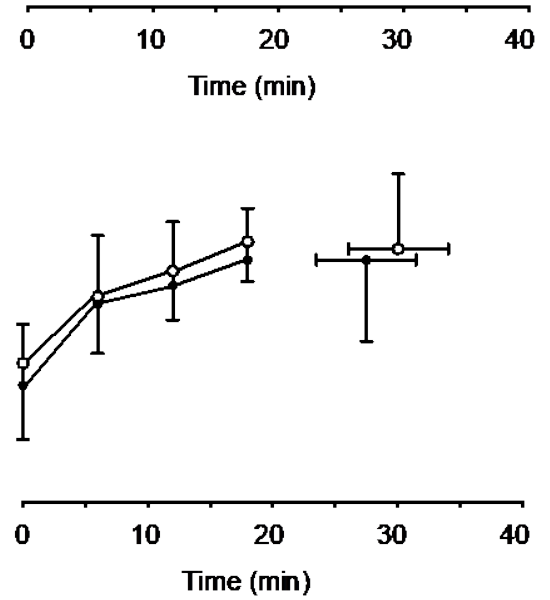
652 Figure 3

653

Male

A

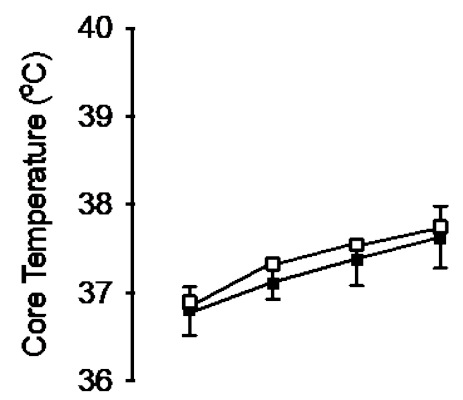

B

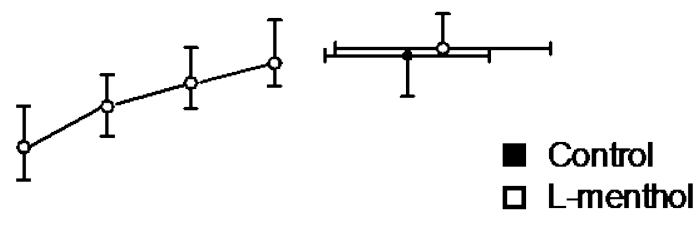

Female

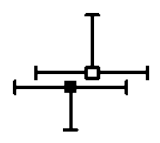

口 L-menthol

654
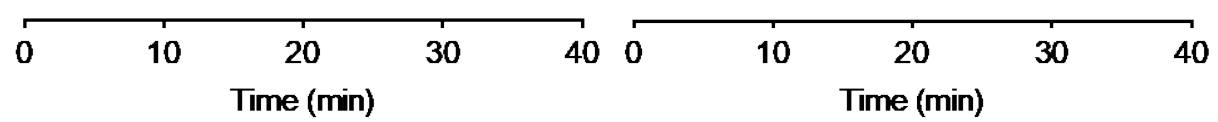

C

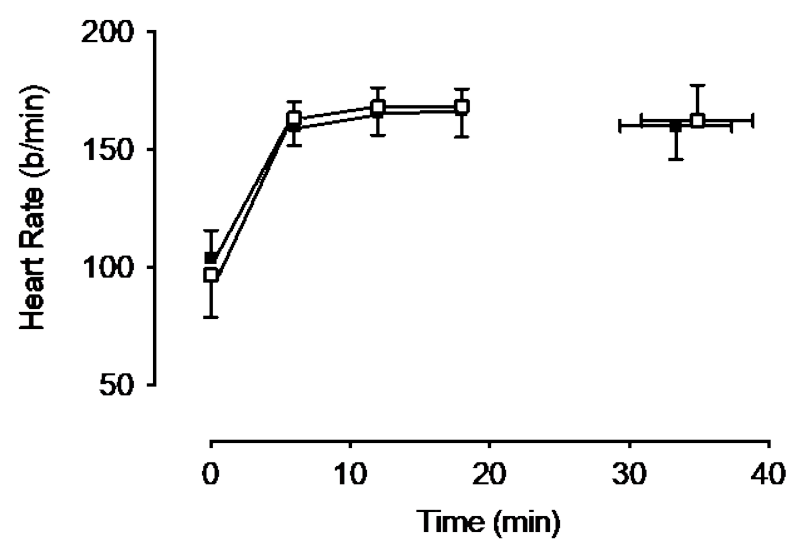

D

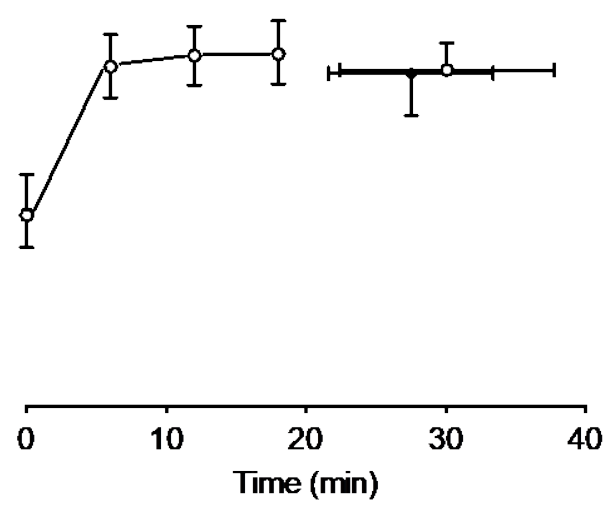

655

656

657

658

659

660

661

662

663 